\title{
EXPONENTIAL-POLYNOMIAL EQUATIONS AND DYNAMICAL RETURN SETS
}

\author{
THOMAS SCANLON AND YU YASUFUKU
}

\begin{abstract}
We show that for each finite sequence of algebraic integers $\alpha_{1}, \ldots, \alpha_{n}$ and polynomials $P_{1}\left(x_{1}, \ldots, x_{n} ; y_{1}, \ldots, y_{n}\right), \ldots, P_{r}\left(x_{1}, \ldots, x_{n} ; y_{1}, \ldots, y_{n}\right)$ with algebraic integer coefficients, there are a natural number $N, n$ commuting endomorphisms $\Phi_{i}: \mathbb{G}_{m}^{N} \rightarrow \mathbb{G}_{m}^{N}$ of the $N^{\text {th }}$ Cartesian power of the multiplicative group, a point $P \in \mathbb{G}_{m}^{N}(\mathbb{Q})$, and an algebraic subgroup $G \leq \mathbb{G}_{m}^{N}$ so that the return set $\left\{\left(\ell_{1}, \ldots, \ell_{n}\right) \in \mathbb{N}^{n}: \Phi_{1}^{\circ \ell_{1}} \circ \cdots \circ \Phi_{n}^{\circ \ell_{n}}(P) \in G(\mathbb{Q})\right\}$ is identical to the set of solutions to the given exponential-polynomial equation: $\left\{\left(\ell_{1}, \ldots, \ell_{n}\right) \in\right.$ $\left.\mathbb{N}^{n}: P_{1}\left(\ell_{1}, \ldots, \ell_{n} ; \alpha_{1}^{\ell_{1}}, \ldots, \alpha_{n}^{\ell_{n}}\right)=\cdots=P_{r}\left(\ell_{1}, \ldots, \ell_{n} ; \alpha_{1}^{\ell_{1}}, \ldots, \alpha_{n}^{\ell_{n}}\right)=0\right\}$.
\end{abstract}

\section{INTRODUCTION}

Motivated by the conclusion of Faltings' Theorem on rational points on subvarieties of abelian varieties, Ghioca, Tucker and Zieve posed the following question (Question 1.6 of [2]) about return sets for finite rank algebraic dynamical systems.

Question 1.1. Let $X$ be a variety defined over $\mathbb{C}$, let $V$ be a closed subvariety of $X$, let $S$ be a finitely generated commutative subsemigroup of $\operatorname{End} X$, and let $\alpha \in X(\mathbb{C})$. Do the following hold?

(a) The intersection $V(\mathbb{C}) \cap \mathcal{O}_{S}(\alpha)$ can be written as $\mathcal{O}_{T}(\alpha)$ where $T$ is the union of finitely many cosets of subsemigroups of $S$.

(b) For any choice of generators $\Phi_{1}, \ldots, \Phi_{r}$ of $S$, let $Z$ be the set of tuples $\left(n_{1}, \ldots, n_{r}\right) \in \mathbb{N}^{r}$ for which $\Phi_{1}^{\circ n_{1}} \circ \cdots \circ \Phi_{r}^{\circ n_{r}}(\alpha) \in V(\mathbb{C})$, where $\Phi_{i}^{\circ n_{i}}$ is the $n_{i}$-fold composition of $\Phi_{i}$; then $Z$ is the union of finitely many sets of the form $z_{i}+\left(G_{i} \cap \mathbb{N}^{r}\right)$, where each $G_{i}$ is a subgroup of $\mathbb{Z}^{r}$ and each $z_{i} \in \mathbb{N}^{r}$.

There are some obvious cases in which Question 1.1 has a negative answer. For example, if $X=\mathbb{A}^{m}$ is the affine $m$-space, $\Phi_{i}: \mathbb{A}^{m} \rightarrow \mathbb{A}^{m}$ is given by $\left(x_{1}, \ldots, x_{n}\right) \mapsto$ $\left(x_{1}, \ldots, x_{i-1}, x_{i}+1, x_{i+1}, \ldots, x_{n}\right), \alpha=(0, \ldots, 0)$ is the origin, and $V \subseteq \mathbb{A}^{n}$ is any variety, then $Z=V(\mathbb{C}) \cap \mathbb{N}^{n}$. As is well-known, the set of natural number points on a variety may be very complicated. For another example, consider the case of $X=\mathbb{A}^{2}, \Psi_{1}(x, y)=(2 x, y), \Psi_{2}(x, y)=(x, y+1), \alpha=(1,0)$, and $Y=\Delta_{\mathbb{A}^{1}}=$ $\{(x, y): x=y\}$. Then $Z=\left\{\left(m, 2^{m}\right): m \in \mathbb{N}\right\}$.

With these examples in mind, one might seek geometric conditions on the algebraic dynamical system $(X, S)$ for which a positive answer to Question 1.1 may be expected. In a companion paper [3], the same authors specialized Question 1.1 to the case that $X=\mathbb{G}_{m}^{g}$ is a power of the multiplicative group and $S$ is a semigroup of algebraic group endomorphisms. Under various hypotheses, for example when the

This collaboration was made possible by NSF grant FRG DMS-0854839. The first author was partially supported by NSF grant DMS-1001556, and the second author by JSPS Grants-in-Aid 23740033 . 
differential of each $\Phi_{i}$ at the origin is diagonalizable, they showed that Question 1.1 has a positive answer, but they constructed two examples for which the return sets are infinite but may be represented as the natural number points on a quadratic curve.

In this note we show that such examples are far from anomalous and that, in fact, every set which may be expressed as the natural number solutions of an exponentialpolynomial equation may be realized as the return set for an algebraic dynamical system on some power of the multiplicative group. We proceed by running the by-now-standard Skolem-Mahler-Lech-Chabauty argument in reverse. That is, we start with some easy linear algebraic calculations showing that if $R$ is any commutative ring with no $\mathbb{Z}$-torsion, then every set of natural numbers solutions to a system of exponential-polynomial equations over $R$ may be realized as the return set for a linear dynamical system over $R$. We pull this result down from rings of integers in number fields to $\mathbb{Z}$ to show that every set of solutions to a system of exponential-polynomial equations over $R$ may be realized as the return set of a linear dynamical system over $\mathbb{Z}$. Exponentiating this last linear dynamical system we obtain the desired algebraic dynamical system on an algebraic torus.

\section{Conventions And statement of main theorem}

We include 0 in the set $\mathbb{N}$ of natural numbers. Our fundamental object of study is the return set for an algebraic dynamical system.

Definition 2.1. Given a set $X$, a finite sequence $\Phi_{1}, \ldots, \Phi_{n}$ of self-maps $\Phi_{i}: X \rightarrow$ $X$, a point $a \in X$, and a subset $Y \subseteq X$, we define the return set to be

$$
E\left(a, \Phi_{1}, \ldots, \Phi_{n}, Y\right):=\left\{\left(\ell_{1}, \ldots, \ell_{n}\right) \in \mathbb{N}^{n}: \Phi_{1}^{\circ \ell_{1}} \circ \cdots \circ \Phi_{n}^{\circ \ell_{n}}(a) \in Y\right\}
$$

Remark 2.2. We shall abuse notation somewhat in the case of algebraic dynamical systems. That is, if $X$ is a scheme over the $\operatorname{ring} R, \Phi_{1}, \ldots, \Phi_{n}$ is a sequence of commuting regular self-maps $\Phi_{i}: X \rightarrow X, Y \subseteq X$ is a subscheme and $a \in X(R)$ is an $R$-valued point of $X$, then we write $E\left(a, \Phi_{1}, \ldots, \Phi_{n}, Y\right)$ for $E\left(a, \Phi_{1}^{R}, \ldots, \Phi_{n}^{R}, Y(R)\right)$.

Our main theorem is that the class of return sets for finitely generated commutative semigroups of algebraic group endomorphisms of algebraic tori coincides with the class of exponential-polynomial sets.

Definition 2.3. Let $R$ be a commutative ring and $n$ a natural number. An $R$ exponential-polynomial function of $n$-variables is a function $f: \mathbb{N}^{n} \rightarrow R$ of the form $\left(\ell_{1}, \ldots, \ell_{n}\right) \mapsto P\left(\ell_{1}, \ldots, \ell_{n} ; \alpha_{1}^{\ell_{1}}, \ldots, \alpha_{m}^{\ell_{1}}, \ldots, \alpha_{1}^{\ell_{n}}, \ldots, \alpha_{m}^{\ell_{n}}\right)$ for some polynomial $P$ in $n(m+1)$ variables over $R$ and elements $\alpha_{1}, \ldots, \alpha_{m} \in R$. By an $R$-exponentialpolynomial set we mean a finite intersection of subsets of $\mathbb{N}^{n}$ defined by the vanishing of an $R$-exponential-polynomial function.

With these definitions in place we may express our main theorem.

Theorem 2.4. Let $\mathcal{O}$ be the ring of all algebraic integers and let $Z \subseteq \mathbb{N}^{n}$ be an $\mathcal{O}$ exponential-polynomial set. Then there are an algebraic torus $X$ over $\mathbb{Q}$, an $n$-tuple of commuting endomorphisms $\Phi_{i}: X \rightarrow X$, a point $P \in X(\mathbb{Q})$, and an algebraic subgroup $Y \leq X$ for which $Z=E\left(P, \Phi_{1}, \ldots, \Phi_{n}, Y\right)$. 


\section{SOME BASIC LEMMATA ON EXPONENTIAL-POLYNOMIALS}

For the remainder of this note, $R$ denotes a commutative ring with no $\mathbb{Z}$-torsion. We write $R_{\mathbb{Q}}:=R \otimes \mathbb{Q}$ and regard $R$ as a subring of $R_{\mathbb{Q}}$.

We shall encode general exponential-polynomial sets by representing their defining equations as linear relations amongst basic generalized monomials, but for the sake of concreteness, we regard exponential polynomials as functions.

Definition 3.1. For $k \in \mathbb{N}$ and $a \in R$ we define $\left(\begin{array}{l}a \\ k\end{array}\right):=\frac{1}{k !} \prod_{i=0}^{k-1}(a-i) \in R_{\mathbb{Q}}$, where $\left(\begin{array}{l}a \\ 0\end{array}\right):=1$ as usual. If $\mathbf{a}:=\left(a_{1}, \ldots, a_{n}\right) \in R^{n}$ is an $n$-tuple of elements of $R$ and $\mathbf{k}=\left(k_{1}, \ldots, k_{n}\right) \in \mathbb{N}^{n}$ is an $n$-tuple of natural numbers, then $\left(\begin{array}{l}\mathbf{a} \\ \mathbf{k}\end{array}\right):=\prod_{i=1}^{n}\left(\begin{array}{l}a_{i} \\ k_{i}\end{array}\right)$ and $\mathbf{a}^{\mathbf{k}}:=\prod_{i=1}^{n} a_{i}^{k_{i}}$. By a basic exponential multinomial over $R$ we mean an $R_{\mathbb{Q}^{-}}$ exponential polynomial of the form $\left(\begin{array}{l}\mathbf{x} \\ \mathbf{k}\end{array}\right) \boldsymbol{\lambda}^{\mathbf{x}}$ for some $\mathbf{k} \in \mathbb{N}^{n}$ and $\boldsymbol{\lambda} \in R^{n}$ where $\mathbf{x}=\left(x_{1}, \ldots, x_{n}\right)$ is the $n$-tuple of standard indeterminates in the polynomial ring $R\left[x_{1}, \ldots, x_{n}\right]$.

Lemma 3.2. Every element of $R\left[x_{1}, \ldots, x_{n}\right]$ can be expressed as an $R$-linear combination of the set $\left\{\left(\begin{array}{l}\mathbf{x} \\ \mathbf{k}\end{array}\right): \mathbf{k} \in \mathbb{N}^{n}\right\}$.

Proof. For each $i$, we prove by induction that $x_{i}^{k}$ for any $k$ is an $\mathbb{Z}$-linear combination of the set $\left\{\left(\begin{array}{c}x_{i} \\ j\end{array}\right): j \in \mathbb{N}\right\}$. Indeed $x_{i}^{0}=1=\left(\begin{array}{c}x_{i} \\ 0\end{array}\right)$. More generally, $x_{i}^{k}-k !\left(\begin{array}{c}x_{i} \\ k\end{array}\right)$ is a polynomial with $\mathbb{Z}$-coefficients of degree less than $k$. So this completes the induction. By expansion, we see that any monomial $\mathbf{x}^{\mathbf{j}}$ is a $\mathbb{Z}$-linear combination of the set $\left\{\left(\begin{array}{l}\mathbf{x} \\ \mathbf{k}\end{array}\right): \mathbf{k} \in \mathbb{N}^{n}\right\}$. By tensoring with $R$, the result follows.

Lemma 3.3. Every $R$-exponential-polynomial function may be expressed as a finite $R$-linear combination of basic exponential multinomials.

Proof. Using the laws of exponents, it is easy to see that every $R$-exponentialpolynomial function may be expressed as a finite $R$-linear combination of exponential polynomial functions of the form $\lambda^{\mathbf{x}} \mathbf{x}^{\mathbf{k}}$. By Lemma 3.2 the monomials $\mathbf{x}^{\mathbf{k}}$ may be expressed as $\mathbb{Z}$-linear combinations of basic multinomials. Distributing the product of the exponential term over the sum, we conclude.

\section{Some linear Algebra}

In this section we carry out some basic linear algebraic computations in the service of our main theorem.

Some notation is in order.

Definition 4.1. For any natural number $n$ and $i \leq n$, we denote by $e_{i, n}$ (written as $e_{i}$ if $n$ is understood) the column vector whose $i^{\text {th }}$ entry is 1 and all of whose other entries are 0 . That is, $e_{1, n}, \ldots, e_{n, n}$ is the standard basis of $\mathbb{Z}^{n}$. The linear map $J_{n}$ is defined by $J_{n} e_{i, n}=e_{i+1, n}$ for $i<n$ and $J_{n} e_{n, n}=0$. That is, considered as an $n \times n$ matrix $J_{n}$ is the element of $M_{n \times n}(\mathbb{Z})$ with 1 s along the subdiagonal and $0 \mathrm{~s}$ in every other entry. If $n$ is understood or otherwise immaterial, we write $J$ for $J_{n}$. We write $I_{n}$ for the identity matrix in $M_{n \times n}$ and again write $I$ if $n$ is understood. For any ring $R$ as there is a unique map $\mathbb{Z} \rightarrow R$, we regard $J_{n}$ and $I_{n}$ as elements of $M_{n \times n}(R)$ and $e_{i, n}$ as an element of $R^{n}$.

Lemma 4.2. For any $n$-tuple $\mathbf{j}=\left(j_{1}, \ldots, j_{n}\right)$ of natural numbers, there is another n-tuple $\mathbf{M}=\left(M_{1}, \ldots, M_{n}\right)$ of natural numbers having the property that the only 
$n$-tuple $\mathbf{k}=\left(k_{1}, \ldots, k_{n}\right)$ of natural numbers satisfying $\mathbf{k} \cdot \mathbf{M}:=\sum_{i=1}^{n} k_{i} M_{i}=\mathbf{j} \cdot \mathbf{M}$ is $\mathbf{k}=\mathbf{j}$.

Proof. Let $p_{1}, \ldots, p_{n}$ be a sequence of distinct primes for which $j_{i}<p_{i}$ for each $i \leq n$. Set $M_{i}:=\prod_{\ell \neq i} p_{\ell}$. If $\mathbf{k} \cdot \mathbf{M}=\mathbf{j} \cdot \mathbf{M}$, then $k_{i} M_{i} \equiv j_{i} M_{i}\left(\bmod p_{i}\right)$ for each $i \leq n$. As $M_{i}$ is a product of primes distinct from the prime $p_{i}$ we conclude that $M_{i}$ is invertible modulo $p_{i}$ so that $k_{i} \equiv j_{i}\left(\bmod p_{i}\right)$. Thus, we may write $k_{i}=j_{i}+\epsilon_{i} p_{i}$ for some integer $\epsilon_{i}$. As $j_{i}<p_{i}$ and $k_{i} \geq 0$, we conclude that $0 \leq k_{i}=j_{i}+\epsilon_{i} p_{i}<\left(1+\epsilon_{i}\right) p_{i}$ so that $\epsilon_{i} \geq 0$. We then have

$$
\mathbf{j} \cdot \mathbf{M}=\mathbf{k} \cdot \mathbf{M}=\sum_{i=1}^{n}\left(j_{i}+\epsilon_{i} p_{i}\right) M_{i}=\mathbf{j} \cdot \mathbf{M}+\left(\sum_{i=1}^{n} \epsilon_{i}\right)\left(\prod_{\ell=1}^{n} p_{\ell}\right) .
$$

Thus, $0=\sum_{i=1}^{n} \epsilon_{i}$. As each $\epsilon_{i}$ is nonnegative, we conclude that they are all equal to zero. That is, $\mathbf{j}=\mathbf{k}$.

Proposition 4.3. For any natural number $n, n$-tuple $\boldsymbol{\lambda} \in R^{n}$ of elements of $R$ and $n$-tuple $\mathbf{j} \in \mathbb{N}^{n}$ of natural numbers, there are some finite rank free $R$-module $F$, an $R$-linear map $\pi: F \rightarrow R$, an element $v$, and an $n$-tuple $\psi_{1}, \ldots, \psi_{n}$ of commuting endomorphisms of $F$ so that for all n-tuples $\boldsymbol{\ell} \in \mathbb{N}^{n}$ of natural numbers one has

$$
\pi \circ \psi_{1}^{\circ \ell_{1}} \circ \cdots \circ \psi_{n}^{\circ \ell_{n}}(v)=\lambda^{\ell}\left(\begin{array}{l}
\ell \\
\mathbf{j}
\end{array}\right)
$$

Proof. Let $\mathbf{M}=\left(M_{1}, \ldots, M_{n}\right) \in \mathbb{N}^{n}$ be the sequence of natural numbers provided by Lemma 4.2. Let $N:=\mathbf{M} \cdot \mathbf{j}+1$ and $F:=R^{N}$, and set $\psi_{i}:=\lambda_{i}\left(I+J^{M_{i}}\right)$. As $\left\{\psi_{1}, \ldots, \psi_{n}\right\} \subseteq R[J]$, the subring of $M_{N \times N}(R)$ they generate is commutative. Using the usual binomial expansions, one computes immediately that for any $\left(\ell_{1}, \ldots, \ell_{n}\right) \in \mathbb{N}^{n}$, one has

$$
\begin{aligned}
\left(\psi_{1}^{\circ \ell_{1}} \circ \cdots \circ \psi_{n}^{\circ \ell_{n}}\right)\left(e_{1, N}\right) & =\left(\prod_{i=1}^{n}\left(\lambda_{i}\left(I+J^{M_{i}}\right)\right)^{\ell_{i}}\right)\left(e_{1, N}\right) \\
& =\sum_{\mathbf{k} \in \mathbb{N}^{n}} \lambda^{\ell}\left(\begin{array}{l}
\ell \\
\mathbf{k}
\end{array}\right) J^{\mathbf{k} \cdot \mathbf{M}}\left(e_{1, N}\right) \\
& =\sum_{m=0}^{N}\left(\sum_{\mathbf{k} \cdot \mathbf{M}=m} \lambda^{\ell}\left(\begin{array}{l}
\boldsymbol{\ell} \\
\mathbf{k}
\end{array}\right)\right) e_{m+1, N}
\end{aligned}
$$

As the only solution to $\mathbf{k} \cdot \mathbf{M}=N-1$ is given by $\mathbf{k}=\mathbf{j}$, we conclude that the coefficient of $e_{N, N}$ in $\psi_{1}^{\circ \ell_{1}} \circ \cdots \circ \psi_{n}^{\circ \ell_{n}}(v)$ is $\boldsymbol{\lambda}^{\ell}\left(\begin{array}{l}\boldsymbol{\ell} \\ \mathbf{j}\end{array}\right)$, where $v:=e_{1, N}$. Let $\pi: F \rightarrow R$ be the projection onto the $N^{\text {th }}$ co-ordinate.

Definition 4.4. For $\mathbf{j}=\left(j_{1}, \ldots, j_{n}\right)$ and $\boldsymbol{\lambda}=\left(\lambda_{1}, \ldots, \lambda_{n}\right) \in R^{n}$ as in Proposition 4.3 we let $\left(F_{\mathbf{j}, \boldsymbol{\lambda}}, \psi_{1 ; \mathbf{j}, \boldsymbol{\lambda}}, \ldots, \psi_{n ; \mathbf{j}, \boldsymbol{\lambda}}, v_{\mathbf{j}, \boldsymbol{\lambda}}, \pi_{\mathbf{j}, \boldsymbol{\lambda}}\right)$ be the module, commuting linear maps, vector and projection map obtained in Proposition 4.3

Theorem 4.5. For any commutative ring $R$ with no $\mathbb{Z}$-torsion and $R$-exponentialpolynomial set $Z \subseteq \mathbb{N}^{n}$ there are a finite-rank free $R$-module $F, n$-tuple $\psi_{1}, \ldots, \psi_{n}$ of commuting $R$-module endomorphisms on $F$, point $a \in F$, and submodule $S \leq F$ for which $Z=E\left(a, \psi_{1}, \ldots, \psi_{n}, S\right)$. Moreover, $S$ may be taken to be the kernel of an $R$-linear map $\theta: F \rightarrow Q$, where $Q$ is also a finite-rank free $R$-module. 
Proof. It suffices to show that the zero set of a single $R$-exponential-polynomial may be encoded as a return set. Indeed, if $\left(F_{i}, \psi_{i, 1}, \ldots, \psi_{i, n}, a_{i}, S_{i}\right)$ has return set $Z_{i}$ for $1 \leq i \leq m$, then

$$
\left(\bigoplus_{i=1}^{m} F_{i}, \bigoplus_{i=1}^{m} \psi_{i, 1}, \ldots, \bigoplus_{i=1}^{m} \psi_{i, n}, \quad\left(a_{1}, \ldots, a_{m}\right), \bigoplus_{i=1}^{m} S_{i}\right)
$$

has return set $\bigcap_{i=1}^{m} Z_{i}$.

Let $Z$ be the zero set of an $R$-exponential-polynomial function $f(\mathbf{x})$. By Lemma 3.3 we may write $f=\sum r_{\mathbf{j}, \boldsymbol{\lambda}} \boldsymbol{\lambda}^{\mathbf{x}}\left(\begin{array}{l}\mathbf{x} \\ \mathbf{j}\end{array}\right)$ where $r_{\mathbf{j}, \boldsymbol{\lambda}} \in R$ and the sum is taken over a finite set $A$ of pairs $(\mathbf{j}, \boldsymbol{\lambda})$ where $\mathbf{j} \in \mathbb{N}^{n}$ and $\boldsymbol{\lambda} \in R^{n}$.

Let $F:=\bigoplus F_{\mathbf{j}, \boldsymbol{\lambda}}, \psi_{i}:=\bigoplus \psi_{i ; \mathbf{j}, \boldsymbol{\lambda}}$ for $i \leq n, a=\oplus v_{\mathbf{j}, \boldsymbol{\lambda}}, Q=R$, and $S$ to be the kernel of the composite $\theta$ of $\bigoplus r_{\mathbf{j}, \boldsymbol{\lambda}} \pi_{\mathbf{j}, \boldsymbol{\lambda}}$ and the sum map, where the direct sum and the sum are over $(\mathbf{j}, \boldsymbol{\lambda}) \in A$.

From Proposition 4.3 for any $\ell \in \mathbb{N}^{n}$ we have $\theta \circ \psi_{1}^{\circ \ell_{1}} \circ \cdots \circ \psi_{n}^{\circ \ell_{n}}(a)=f(\ell)$. Thus, $Z=E\left(a, \psi_{1}, \ldots, \psi_{n}, S\right)$ as claimed.

\section{RETURN SETS ON TORI}

In this section we deduce Theorem 2.4 from the linear algebraic Theorem 4.5 .

Throughout this section we denote by $\mathcal{O}$ the ring of all algebraic integers.

Let us note first that every exponential-polynomial set over the algebraic integers may be realized by a linear dynamical system over $\mathbb{Z}$.

Proposition 5.1. If $Z \subseteq \mathbb{N}^{n}$ is a $\mathcal{O}$-exponential-polynomial set, then there are natural numbers $r$ and $s$, a sequence $\phi_{1}, \ldots, \phi_{n}$ of commuting $r \times r$-matrices over $\mathbb{Z}$, a vector $\mathbf{a} \in \mathbb{Z}^{r}$, and a $\mathbb{Z}$-module map $L: \mathbb{Z}^{r} \rightarrow \mathbb{Z}^{s}$ so that if $T:=\operatorname{ker} L$, then $Z=\left\{\ell \in \mathbb{N}^{n}: \phi_{1}^{\circ \ell_{1}} \cdots \phi_{n}^{\circ \ell_{n}}(\mathbf{a}) \in T\right\}$.

Proof. Let $R$ be the subring of $\mathcal{O}$ generated by the bases of the exponents appearing in the expressions of some finite list of $\mathcal{O}$-exponential-polynomial functions whose zero set is equal to $Z$. As each such number is integral over $\mathbb{Z}, R$ is free of finiterank as a $\mathbb{Z}$-module. Let $\left(F, \psi_{1}, \ldots, \psi_{n}, a, S\right)$ be given by Theorem 4.5 for $R$ and $Z$ and let $\theta: F \rightarrow Q$ be an $R$-linear map with $\operatorname{ker} \theta=S$. Then $F$ is also a finite rank free $\mathbb{Z}$-module and all of the listed maps are $\mathbb{Z}$-linear. Choosing a basis, we may identify $F$ with $\mathbb{Z}^{r}$ and each $\psi_{i}$ with some $r \times r$ matrix $\phi_{i}$. Likewise, fixing a $\mathbb{Z}$-basis for $Q$, we may regard $\theta$ as an $s \times r$ matrix. As the dynamical systems $\left(F, \psi_{1}, \ldots, \psi_{n}\right)$ and $\left(\mathbb{Z}^{r}, \phi_{1}, \ldots, \phi_{n}\right)$ (after a choice of basis) are identical as are the initial points and target sets, their return sets are identical.

Finally, let us finish the proof of the main theorem.

Proof of Theorem 2.4. Let $Z \subseteq \mathbb{N}^{n}$ be any $\mathcal{O}$-exponential-polynomial set. Let $r, s$, $L, T$, a, and $\phi_{1}, \ldots, \phi_{n}$ be given by Proposition 5.1 . Since $\operatorname{End}\left(\mathbb{G}_{m}\right)=\mathbb{Z}$, we may identify $\operatorname{Hom}\left(\mathbb{G}_{m}^{r}, \mathbb{G}_{m}^{s}\right)$ with $M_{s \times r}(\mathbb{Z})$ and $\operatorname{End}\left(\mathbb{G}_{m}^{r}\right)$ with $M_{r \times r}(\mathbb{Z})$. Let $\Phi_{i}: X \rightarrow$ $X$ be the endomorphism of $\mathbb{G}_{m}^{r}$ corresponding to $\phi_{i}$ under this identification and let $Y$ be the kernel of the map corresponding to $L$. Let $P:=\left(2^{a_{1}}, \ldots, 2^{a_{r}}\right)$ where the $a_{i}$ s are the components of $\mathbf{a}$. Since 2 has infinite order, $E\left(P, \Phi_{1}, \ldots, \Phi_{n}, Y\right)=$ $E\left(\mathbf{a}, \phi_{1}, \ldots, \phi_{n}, T\right)=Z$. 


\section{Concluding Remarks}

We end this note with a few observations, an explicit example, and some open questions.

Remark 6.1. If $X$ is a semiabelian variety over a field $K$ of characteristic zero, $\Phi_{1}, \ldots, \Phi_{n}$ is a finite sequence of commuting endomorphisms of $X, Y \subseteq X$ is a subvariety, and $a \in X(K)$ is any point, then the return set $E\left(a, \Phi_{1}, \ldots, \Phi_{n}, Y\right)$ is necessarily an $\mathcal{O}$-exponential-polynomial set. Indeed, this result follows from the Mordell-Lang conjecture (or theorem of Faltings and Vojta) and the SkolemMahler-Lech-Chabauty method and is implicit in 3. Our Theorem 2.4 generalizes immediately to the case that $X$ is taken to be a power of a semiabelian variety instead of $\mathbb{G}_{m}$. Thus, Theorem 2.4 may be read as saying that the class of return sets for actions of finitely generated commutative monoids on semiabelian varieties over fields of characteristic zero is precisely the class of $\mathcal{O}$-exponential-polynomial sets.

Remark 6.2. We have stated Theorem 2.4 as an identity of point sets, but as the reader will see from from the proof, we actually convert a system of defining equations for an $\mathcal{O}$-exponential-polynomial system into an algebraic dynamical system with a fixed starting point and target set so that the problem of membership in the return set is reducible to the corresponding problem of solving the given exponential-polynomial equations. That is, these problems are computationally equivalent.

Example 6.3. Our method of construction is effective. For example, let $f\left(\ell_{1}, \ell_{2}\right)=$ $(1+\sqrt{2})^{\ell_{1}} \ell_{1} \ell_{2}-21 \ell_{2}^{2}-5 \sqrt{2} \ell_{1}$, whose zeroes include $(3,1)$. Using binomials, $f\left(\ell_{1}, \ell_{2}\right)=(1+\sqrt{2})^{\ell_{1}} \ell_{1} \ell_{2}-42\left(\begin{array}{c}\ell_{2} \\ 2\end{array}\right)-21 \ell_{2}-5 \sqrt{2} \ell_{1}$. Let $R=\mathbb{Z}[\sqrt{2}]$. We can actually use $\mathbf{M}=(3,2)$ for each of the four terms, so $\psi_{2}$ is $I+J^{2}$ for each of the four blocks and $\psi_{1}$ is $(1+\sqrt{2})\left(I+J^{3}\right)$ for the first block and $\left(I+J^{3}\right)$ for the last three blocks. The sizes of the blocks are $\mathbf{j} \cdot \mathbf{M}+1$, so they are $6,5,3$, and 4 in order. Letting $\mathbf{a}$ be the vector which is 1 in $x_{1}, x_{7}, x_{12}$, and $x_{15}$ and 0 everywhere else, the condition that $\psi_{1}^{\circ \ell_{1}} \circ \psi_{2}^{\circ \ell_{2}}(\mathbf{a})$ is in the set defined by $x_{6}-42 x_{11}-21 x_{14}-5 \sqrt{2} x_{18}=0$ is precisely $f\left(\ell_{1}, \ell_{2}\right)$. Finally, let $x_{i}=y_{i}+z_{i} \sqrt{2}$ and exponentiate: the first coordinate of $\psi_{1}\left(x_{1}, \ldots, x_{18}\right)$ is $(1+\sqrt{2}) x_{1}=\left(y_{1}+2 z_{1}\right)+\left(y_{1}+z_{1}\right) \sqrt{2}$, so the first two coordinates of $\Phi_{1}\left(Y_{1}, Z_{1}, \ldots, Y_{18}, Z_{18}\right)$ are $Y_{1} Z_{1}^{2}$ and $Y_{1} Z_{1}$ and we continue in the same manner to construct $\Phi_{1}$ and $\Phi_{2}$. Here, $P$ is the point which is 2 at $Y_{1}, Y_{7}, Y_{12}$, and $Y_{15}$ and 1 everywhere else, and the subgroup $Y$ is defined by $Y_{6}=Y_{11}^{42} Y_{14}^{21} Z_{18}^{10}$ and $Z_{6}=Y_{18}^{5}$.

Remark 6.4. As is clear from Example6.3, our construction in proving Theorem 2.4 does not optimize the dimension of the algebraic torus on which our dynamical system acts. In fact, any $\mathbb{Z}$-linear function $r_{1} \ell_{1}+r_{2} \ell_{2}$ can be achieved by a $2 \times 2$ block: $\psi_{1}=I+r_{1} J, \psi_{2}=I+r_{2} J$. It remains an open question to determine the minimum dimension of $\mathbb{G}_{m}^{N}$ on which we can generate all of the exponential-polynomial functions of a fixed degree, where the degree of an exponential-polynomial function $P\left(\ell_{1}, \ldots, \ell_{n} ; \alpha_{1}^{\ell_{1}}, \ldots, \alpha_{m}^{\ell_{1}}, \ldots, \alpha_{1}^{\ell_{n}}, \ldots, \alpha_{m}^{\ell_{n}}\right)$ is defined to be the degree in the first $n$ variables.

Remark 6.5. It follows from our Theorem 2.4 and the work of Davis, Putnam and Robinson [1] on the representability of recursively enumerable sets as exponential diophantine sets, that many natural questions about algebraic dynamics 
are undecidable. For example, there is no algorithm which takes as input a tuple of the form $\left(N, \Phi_{1}, \ldots, \Phi_{n+1}, m, a, T\right)$ where $n, m$, and $N$ are natural numbers, $\Phi_{i}: \mathbb{G}_{m}^{N} \rightarrow \mathbb{G}_{m}^{N}$ are commuting endomorphisms, $T \leq \mathbb{G}_{m}^{N}, a \in \mathbb{G}_{m}^{N}(\mathbb{Q})$ and answers correctly whether or not there is an $n$-tuple $\left(\ell_{1}, \ldots, \ell_{n}\right) \in \mathbb{N}^{n}$ with $\Phi_{1}^{\circ \ell_{1}} \circ \cdots \circ \Phi_{n}^{\circ \ell_{n}} \circ \Phi_{n+1}^{\circ m}(a) \in T(\mathbb{Q})$.

\section{REFERENCES}

[1] Martin Davis, Hilary Putnam, and Julia Robinson, The decision problem for exponential Diophantine equations, Ann. of Math., 74 no. 3 (1961), 425 - 436.

[2] Dragos Ghioca, Thomas J. Tucker, and Michael E. Zieve, Linear relations between polynomial orbits, Duke Math. J. 161 (2012), no. 7, 1379 - 1410.

[3] Dragos Ghioca, Thomas J. Tucker, and Michael E. Zieve, The Mordell-Lang question for endomorphisms of semiabelian varieties, J. Théor. Nombres Bordeaux 23 (2011), no. 3, 645 -666 .

University of California, Berkeley, Department of Mathematics, Evans Hall, BerkeLEY, CA 94720-3840, USA

E-mail address: scanlon@math.berkeley.edu

Nihon University, College of Science and Technology, Department of Mathemtaics, 1-8-14 Kanda-Surugadai, ChIYOdA, TOKYo 101-8308, JAPAN

E-mail address: yasufuku@math.cst.nihon-u.ac.jp 